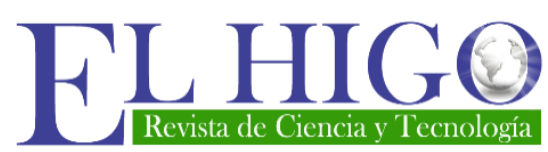

Vol. 06. pp. 02-13/Diciembre 2016

\title{
ELEMENTO BASE PARA LA SOSTENIBILIDAD DEL APROVECHAMIENTO DE RESIDUOS SÓLIDOS MUNICIPALES DE ESTELÍ.
}

\section{BASE ELEMENT FOR THE SUSTAINABILITY OF THE USE OF ESTELÍ MUNICIPAL SOLID WASTE.}

\author{
Henry Javier Vílchez Pérez ${ }^{1}$
}

RESUMEN: Este trabajo tuvo como propósito establecer estrategias que guíen el desarrollo de la formulación de un proyecto de planta de Digestión Anaerobia para tratar la Fracción Orgánica de los Residuos Sólidos Urbanos (FORSU) de Estelí, que sea factible de implementar por la municipalidad. Este proyecto pretenden dar solución, o bien, mitigar, las diferentes problemáticas ambientales que se derivan del manejo inadecuado de residuos sólidos orgánicos; así como también, plantear alternativas para el aprovechamiento económico y energético de los productos obtenidos de dicho tratamiento.

PALABRAS CLAVES: Digestión anaerobia; residuos sólidos urbanos; mitigar; alternativas.

ABSTRACT: This work is dedicated to establishing strategies that will guide the development of the formulation of an Anaerobic Digestion plant project to treat the Organic Fraction of Urban Solid Waste (FORSU) of Estelí, which is feasible to implement by the municipality. This project aims to solve or mitigate the different environmental problems that arise from the inadequate management of organic solid waste; As well as to propose alternatives for the economic and energy use of the products obtained from such treatment.

KEYWORDS: Anaerobic digestion; Urban solid waste; to mitigate; alternatives.

\section{INTRODUCCIÓN}

El municipio de Estelí presenta grandes problemas con el manejo de sus residuos sólidos, siendo uno de los centros urbanos más grandes de Nicaragua con una población de 147,024 personas (INIDE, 2014). Hoy en día no cuenta una gestión de residuos en etapa temprana, la cual es deficiente para el manejo de las 124.47 toneladas diarias que se producen (Vilches \& Moraga, 2010). Actualmente sólo un $13 \%$ de los residuos sólidos urbanos (RSU) son reciclados o reutilizados (AME, 2009).

De acuerdo a Vílchez \& Moraga (2010), en el estudio de caracterización de residuos sólidos domiciliares el $60 \%$ de los RSU es fracción orgánica. Esta fracción se denomina Fracción

\footnotetext{
${ }^{1}$ Docente/Investigador del Programa de Investigación, Estudios Nacionales y Servicios Ambientales, PIENSA Universidad Nacional de Ingeniería, UNI jvil2p@gmail.com o Henry.Vilchez@piensa.uni.edu.ni Apartado Postal: 5595
} 
orgánica de los residuos sólidos urbanos (FORSU). No obstante, esta fracción de la FORSU es desaprovechada por la ciudad de Estelí.

La digestión anaerobia se ha convertido en una tecnología establecida y probada para el tratamiento de residuos sólidos, procedentes de los residuos sólidos orgánicos, mercado y otros compuestos orgánicos industriales (De Baere, 2000). En esta digestión se produce gas combustible, el cual tiene una alta concentración de metano $\left(\mathrm{CH}_{4}\right)$ (aproximadamente $60 \%$ ), con una capacidad calorífica de $5,500 \mathrm{kcal} / \mathrm{m} 3$. Este gas se le denomina comúnmente biogás (Elías, 2012).

Este combustible tiene un valor económico superior a la composta, además de presentarse como una alternativa a la problemática de los crecientes aumentos en los precios de los combustibles.

La digestión anaerobia de residuos sólidos orgánicos tiene una gran aceptación y aplicación en el continente europeo, siendo países como Alemania, Suecia, Noruega, Finlandia, Suiza y Holanda grandes desarrolladores de esta tecnología (Nayono, 2009). Entre las tecnologías comerciales se encuentran: Kompogas, BTA, Waasa, Colsen, Cambi, entre otros.

Por otro lado actualmente se está emprendiendo en México y Colombia la construcción de plantas que tratan la FORSU por un proceso digestión anaerobia el cual consiste en degradar la materia en ausencia de oxigeno por microorganismo, obteniéndose un gas que se puede aprovechar.

No obstante, el caso nicaragüense difiere al europeo, mexicano y colombiano, pues no existe en el país una tecnología a escala industrial, a excepción de la recuperación de biogás en rellenos sanitarios con ayuda del llamado "Modelo del Biogás Centro Americano" desarrollado por la United States Enviromental Protection Agency (US EPA, por sus siglas en inglés) (US EPA, 2013).

Es por esto que es imprescindible en Nicaragua desarrollar la tecnología de digestión anaerobia a nivel industrial para poder dar un manejo sustentable a los residuos y obtener una ganancia energética.

En Nicaragua, no se tiene experiencia en la construcción de plantas de digestión anaerobia a nivel municipal para el tratamiento, valorización y disposición de los residuos sólidos orgánicos urbanos, por lo tanto no existe información que respalde, promueva o apoye un proyecto para incentivar la construcción de una planta de estas características en la ciudad de Estelí.

En este artículo se plantea estrategias que guíen el desarrollo de la formación de una propuesta de proyecto de planta de digestión anaerobia para tratar la FORSU que sea factible de implementar por parte de la municipalidad de Estelí. Estos proyectos pretenden dar solución o bien mitigar las diferentes problemáticas ambientales que se derivan del manejo inadecuado de residuos sólidos orgánicos. 


\section{METODOLOGÍA}

Tras una revisión de la información bibliográfica de los fundamentos teóricos en lo que se describe la situación en torno a la generación y manejo de residuos en Estelí así como una revisión de las experiencia en tecnologías de Digestión Anaerobia (DA), conceptos estratégicos y proyectos de inversión, así como el marco legal y político en torno a los residuos en Nicaragua.

Se elaboró un análisis en el que se determina el problema que enfrentaría el desarrollo de una planta de DA, estableciendo las barreras que impedirían el desarrollo de esta tecnología pero también se discuten algunas alternativas que podrán solucionar las barreras, ver figura 1.

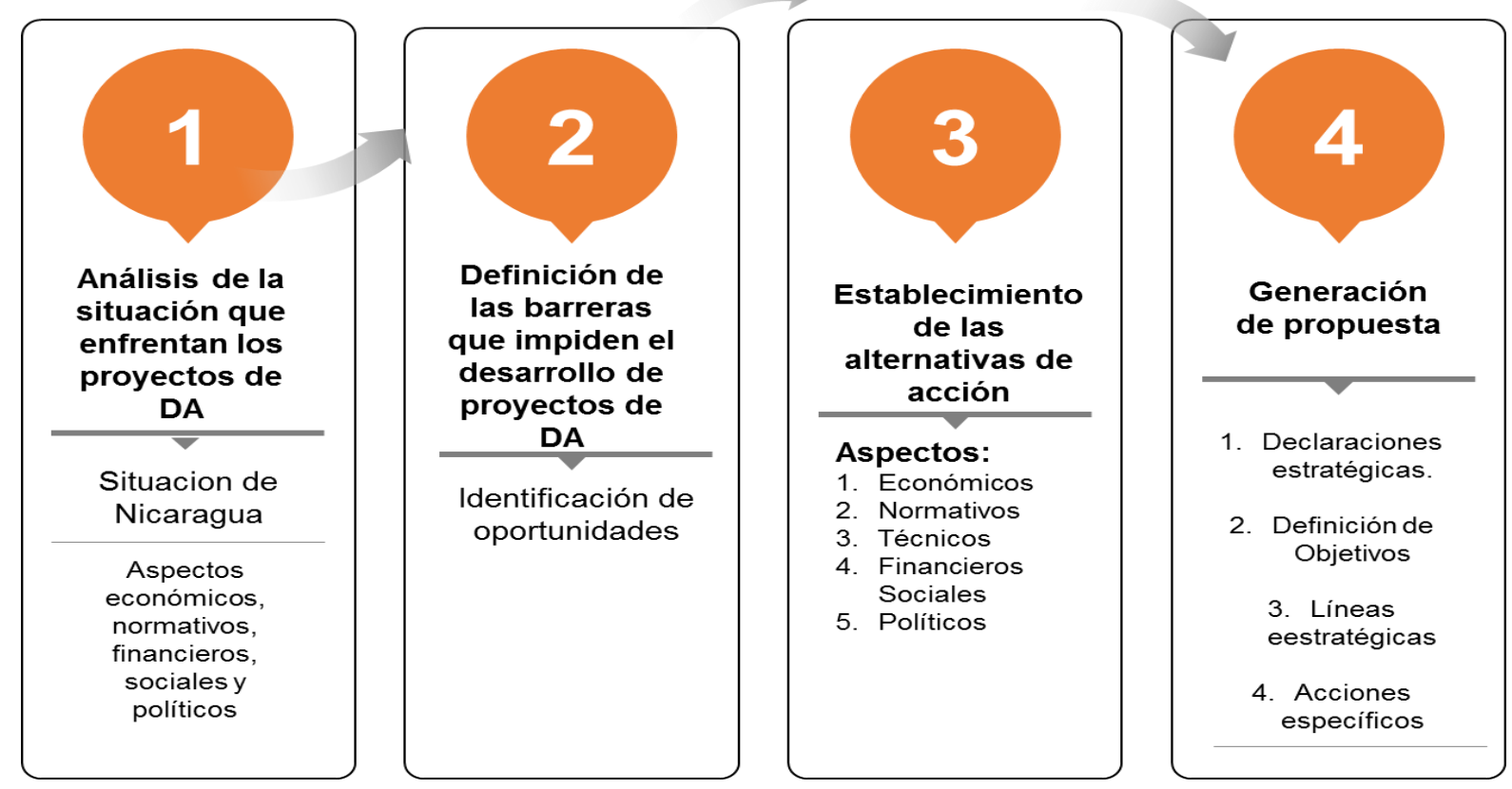

Figura 1: Metodología propuesta para el desarrollo de una planta de DA

\section{RESULTADOS Y DISCUSIÓN}

\section{Análisis de la situación que enfrentan los proyectos de DA en Nicaragua}

Situación en Nicaragua, aspectos económicos, técnicos, normativos, financieros, sociales y políticos.

El empleo de la DA aplicada a la FORSU es una tecnología inexistente en Nicaragua, no se cuenta con normas y especificaciones precisas con las que deba cumplir el proceso, salvo las mismas con las que deba cumplir los sitios de tratamiento y disposición final de residuos sólidos como rellenos sanitarios.

Aunque con la aprobación de la Ley Especial de Gestión Integral de Residuos y Desechos Sólidos Peligrosos y no Peligrosos" y su reglamento, generó en nuestro País, un hito importante 
para la gestión ambiental y el saneamiento, debido a que se establecieron los derechos, obligaciones, atribuciones y responsabilidades de la sociedad, para asegurar una gestión de residuos sólidos sanitaria y ambientalmente adecuada, tomando en cuenta diversos principios como el de minimización, prevención de riesgos ambientales y sobretodo la protección de la salud y el bienestar humano.

Por lo que también plantea una disposición inclinada a promover la implementación y desarrollo de nuevas tecnologías que mejoren el manejo y gestión actual de los residuos así como ayuden a mitigar los efectos nocivos al ambiente

En lo que respecta a los aspectos financieros, desde el punto de vista de negocio dentro de la gestión de residuos, el empleo de la DA únicamente para dar tratamiento a la FORSU no resulta rentable puesto que al igual que todas las tecnologías de disposición final requieren de grandes inversiones y los costos de operación son muy altos la implementación de estos proyectos en el país han sido promovidos por ONGs, para los cuales de acuerdo a estudios elaborados por Jacotin (2010), la TIR requerida debe ser de por lo menos del $12 \%$, lo cual nunca ha sido alcanzada en el país.

Los montos de inversión son muy elevados y al ser visto únicamente como negocio en la gestión de residuos, los proyectos de DA contemplan muy pocos beneficios sociales, teniendo una situación desfavorable frente a otros proyectos o programas de inversión que también requieren financiamiento de los recursos del estado.

En contexto con este panorama, existen diversos programas con fondos para dar financiamiento mediante subvenciones y préstamos a proyectos con características de infraestructura social, sustentabilidad, energía renovables y ambientales como es el caso de los proyectos mediante DA.

\section{Barreras que impiden el desarrollo de proyectos de DA}

Las principales barreas a las que se enfrentara el proyecto será:

Barrera económica: los costos de inversión son muy elevados así como los costos de operación de la planta al requerir de un personal calificado respecto a los costos de operación instalación de un relleno sanitario, en los que el personal es de menor capacitación.

Costos por tratamiento y disposición mayores a los de los rellenos sanitarios debido al costo integral del proceso.

Costos por pretratamiento de los residuos requeridos al tratarse de residuos generados en composición mixta por lo que se le adicional el costo de la separación de los residuos productos en la ciudad no se implementa la separación selectiva de los residuos en los hogares. 
- Barrera técnica: es una tecnología cuya aplicación a RSU es inexistente en Nicaragua, por lo que no se cuenta con experiencia en el desarrollo e implementación, así como en lo que corresponde al tratamiento de residuos generados en el país.

Requiere de una segregación al interno de los hogares en residuos degradables y no degradables por lo que la FIRSU se dejan de lado. Su condicionamiento esta en las características físicas y químicas de los residuos.

- Barrera financiera: un de los problemas es que se requiere de elevados montos de inversión, por lo que no es rentable desde el punto de vista de negocio para disposición final de residuos. Los montos de inversión en los programas de inversión anual de las municipalidades son limitados por los beneficios que presenten y por la existencia de otros proyectos que también requieran financiamiento.

La tasa de retorno de inversión es baja, respecto a los requerimientos para inversiones con recursos de las municipalidades.

- Barrera social: se presenta una cultura generalizada al consumismo que desenlaza en una generación de residuos per cápita mayor cada año.

Se tiene un rechazo por parte de los pobladores hacia la implementación de instalaciones de tratamiento de residuos en zonas próximas a las comunidades por motivos de malos olores, emociones y posibles afectaciones a la salud.

Desconocimiento de las consecuencias de manejo de residuos y falta de interés respecto al mismo. Se tiene un desconocimiento generalizados sobre otras técnicas de dispocision final por lo tanto no se conoce sobre los beneficios del tratamiento de residuos a través de DA.

- Barrera de la normativa: En lo que respecta al cuadro normativo, no se trata propiamente de una barrera puesto que al ser, la DA aplicada a la FORSU, una tecnología inexistente en Nicaragua, no se cuenta con normas y especificaciones precisas con las que se deba cumplir el proceso, salvo la Norma Técnica Para El Control Ambiental De Los Rellenos Sanitarios Para Desechos Sólidos No Peligrosos (NTON 05 013 - 01 ) y Norma Técnica Ambiental Para El Manejo, Tratamiento Y Disposición Final De Los Desechos Sólidos No-Peligrosos (NTON 05 014-01).

De tal manera que la falta de normas que regulen las plantas y procesos de DA aunando a la pobre legislación sobre rellenos sanitarios genera una situación desfavorable para proyectos de DA.

- Barreras cruzadas: Factores cruzados de diferentes barreras influyen directa e indirectamente incrementando el efecto negativo sobre el objetivo de desarrollar proyectos de planta de DA. Las barreras económicas están íntimamente relacionadas a las barreras técnicas y estas a su vez a las barreras sociales debido a que se crea una 
cadena consecuencial en la que las condiciones de generación de residuos impactan directamente en la condiciones y requerimientos en las que debe diseñarse el proceso DA, esto impacta directamente en los costos por requerimiento de equipos y de proceso lo cual aumenta los costos de tratamiento.

\section{Oportunidades}

Se encuentran oportunidades de desarrollo en torno a los proyectos de DA que, de aprovecharse, implican cambios en el concepto y desarrollo del proyecto, en su modelo de negocio, en los beneficios que representaría a la sociedad y en la viabilidad de su implementación. Tales oportunidades se encuentran en los marcos normativos, financieros y modelo de negocio.

Ante la problemática derivada del manejo de residuos y operación de los sitios de disposición final, en especial de rellenos sanitarios, el tratamiento de la FORSU mediante DA se presenta no sólo como necesidad sino como oportunidad de negocio debido a la necesidad de implementar más y nuevos sitios de disposición final.

Otra gran oportunidad de negocio consiste en el aprovechamiento de los productos de la digestión anaerobia mediante su comercialización. El biogás tiene potencial de aprovechamiento en lo que respecta a su uso como combustible de vehículos, en generación de electricidad y puede también ser inyectado en la red de gas natural.

El digestato tiene potencial de aprovechamiento en el mercado de fertilizantes. Los mercados en los que pueden incursionar los productos de la DA son mercados que existen en Estelí y que bajo una adecuada estrategia pueden ser explotados. Establecer modelos de negocio aprovechando el digestato y biogás producidos por la DA permite elevar la rentabilidad de sus proyectos así como incrementar los beneficios ambientales y reducir el volumen de residuos enviados a disposición final.

\section{Establecimiento de alternativas}

Para poder establecer alternativas de acción que permitan el desarrollo de proyectos de plantas de DA, es necesario partir de la premisa de que el tratamiento de residuos mediante DA no es una alternativa equivalente a la disposición de residuos que se lleva a cabo en los rellenos sanitarios; puesto que trata únicamente la FORSU y, a diferencia de otras tecnologías, genera productos que tienen un potencial valorizable en diferentes mercados, el proceso de DA estabiliza y reduce el volumen de los residuos que serían enviados a disposición final, sin embargo el aprovechamiento del digestato generado reduce aún más el volumen de residuos enviados a disposición final.

Se proponen las siguientes alternativas de cara a la situación que enfrentan los proyectos de plantas de DA para tratamiento de la FORSU en Nicaragua:

Crear un modelo de negocio del proyecto que considere alternativas de comercialización y aprovechamiento de los productos del proceso de DA. 
Obtención de recursos a través de programas de financiamiento nacional o internacional.

Implementación de políticas a seguir que, buscando una mejor condición ambiental, generen condiciones que propicien el desarrollo de proyectos de plantas de DA.

Implementación de normas de calidad y estándares aplicables a plantas de DA exitosas de otros países cuyas características de proceso sean similares a las de proyectos de plantas de DA a desarrollar en Estelí.

- Modelo de Negocio integral Se propone un modelo de negocio que plantea un cambio de enfoque, en el cual no solamente se pretende dar tratamiento a la FORSU mediante el proceso de DA sino también se busca aprovechar los productos de dicho proceso, otorgando beneficios económicos, energéticos y ambientales.

La Figura 2, muestra un esquema del modelo de negocio integral propuesto para proyectos de plantas de DA que den tratamiento a la FORSU.

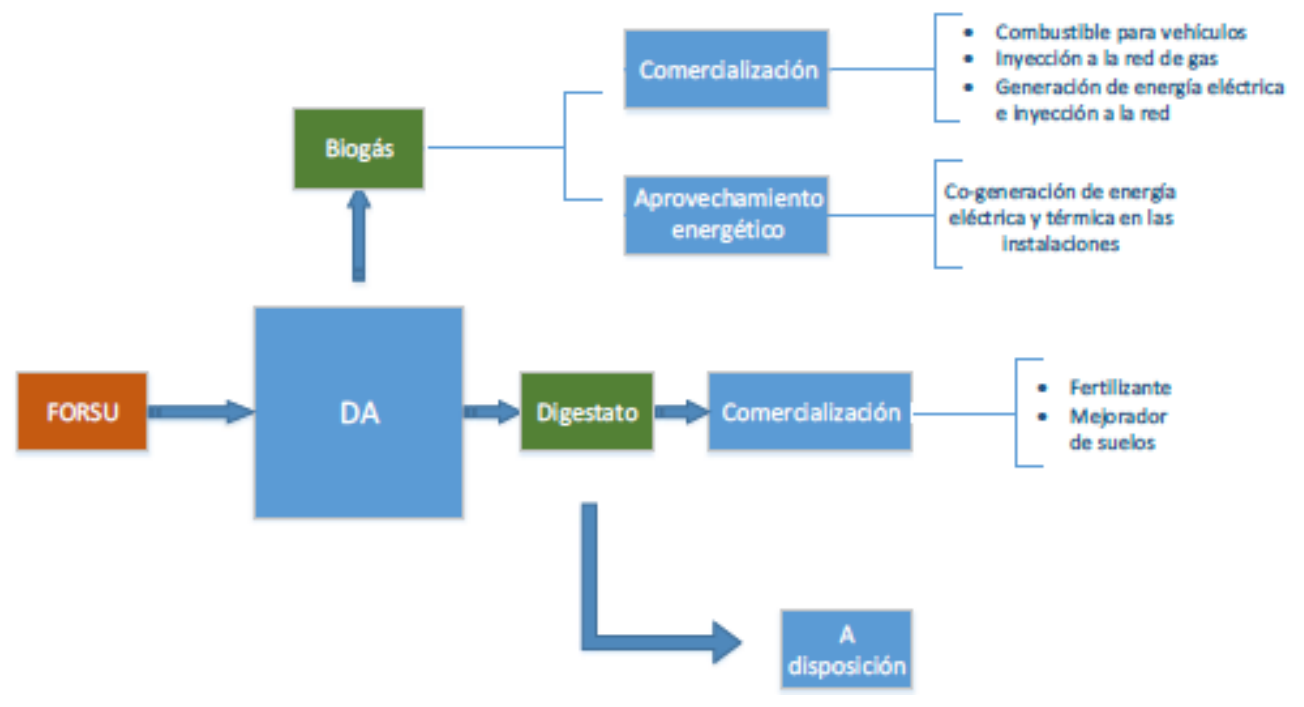

La Figura 2: Esquema del modelo de negocio integral de DA

Fuente: Duran, 2013

Las vías de comercialización del digestato son comercializarlo como fertilizante y como mejorador de suelo.

Las vías de comercialización del biogás son venderlo como combustible para vehículos, venta para inyección a la red de gas natural y venta de energía eléctrica producida por su combustión. Así también, una vía de aprovechamiento es su uso para co-generación de energía eléctrica para consumo en las instalaciones del proyecto. 
- Financiamiento a través de instituciones financieras internacionales: Existen diversas instituciones a nivel internacional cuyo interés es impulsar el crecimiento en infraestructura de países en desarrollo así como impulsar proyectos que ayuden a mitigar o presenten soluciones a problemáticas ambientales, como lo son reducción emisiones, creación de nuevas tecnologías verdes, aprovechamiento de energías renovables, entre otros.

A continuación se presenta una lista de instituciones internacionales que proporcionan diversas alternativas de financiamiento a proyectos de inversión en Nicaragua que se enmarcan en dentro de sus objetivos.

Banco de Desarrollo de América Latina (CAF): Financiamiento vía préstamos, líneas de crédito, participaciones accionarias, financiamiento estructurado.

Concepto del financiamiento: movilización de recursos para la prestación oportuna de servicios financieros múltiples, de alto valor agregado, a clientes de los sectores públicos y privado de los países accionistas.

\section{Banco Interamericano de Desarrollo}

Fondo Multilateral de Inversiones

Financiamiento a través de préstamos, donaciones, garantías e inversiones.

Concepto del financiamiento: soluciones financieras flexibles a sus países miembros para financiar el desarrollo económico y social a través de préstamos y donaciones a entidades públicas y privadas en América Latina y el Caribe, así como programas nacionales y regionales de cooperación técnica en diversas áreas.

\section{Propuesta de estrategia}

Los proyectos de DA son flexibles y por lo tanto la estrategia también debe ser flexible; su desarrollo debe ser adecuado a los requerimientos de cada proyecto y a las condiciones específicas de la zona donde se pretenda implementar siendo, entonces, específico para cada región, por lo que se definen criterios y objetivos mínimos con características generales que pretenden facilitar la superación de las barreras que impiden su desarrollo y generar condiciones a través de acciones propuestas que eleven la probabilidad de éxito de su implementación; sin embargo los objetivos y aspectos de la estrategia pueden y deben ser adecuados a las condiciones de cada proyecto a la vez que es posible agregar otros criterios y acciones que aporten beneficios al desarrollo de la estrategia para implementar proyectos de DA en Estelí.

La propuesta de estrategia se sustenta por una parte en aumentar la factibilidad económica de los proyectos de DA mediante el diseño e implementación de un modelo de negocio general en el que se eleven los flujos de beneficios económicos, en el cual se plantea como entradas el tratamiento de la FORSU y la comercialización de los productos de la DA; por otra parte la estrategia busca aprovechar las oportunidades y superar las barreras presentes en los rubros 
técnico, financiero, social, económico, normativo y político en torno al desarrollo de los proyectos de DA en Estelí.

Por lo anterior, la elaboración de la propuesta de estrategia se realizó partiendo de la definición de elementos de los objetivos del proyecto y siguiendo a sus elementos subsecuentes como niveles inferiores hasta llegar a las acciones específicas, que son en sí los elementos que definen la estrategia.

La propuesta de estrategia se establece para tomar lugar en las fases de formulación del proyecto "Estudio de gran visión" y de "Estudio de pre-factibilidad" puesto que es en estas fases donde se define la problemática a resolver por el proyecto y, sobre todo, se define el modelo de proyecto a implementar.

Las condiciones que pueden diferir en cada proyecto son las siguientes:

Cantidades generadas de residuos, Demanda y porcentaje de residuos generado que se pretenda tratar (capacidad de tratamiento), Características de los residuos (composición mixta, composición, $\mathrm{pH}$, humedad, nutrientes, potencial de generación de biogás), Características de los productos (composición de biogás como el contenido de metano, humedad, contaminantes, etc., y características físicas y químicas del digestato como lo son la relación de nutrientes, contaminantes, composición, humedad, pH, entre otros), Población objetivo Área de influencia, Partes interesadas y afectadas.

\section{Opciones de comercialización y aprovechamiento de productos de los proyectos de plantas de DA}

Los productos del proceso de la digestión anaerobia son el biogás y el digestato, ambos valorizables; las opciones de comercialización y aprovechamiento se presentan a continuación.

\section{Opciones de aprovechamiento del biogás}

- Aprovechamiento energético: mediante producción de energía eléctrica en las instalaciones del proyecto.

- Aprovechamiento económico mediante su comercialización como combustible para vehículos.

- Aprovechamiento económico mediante su venta para inyección a la red de gas natural.

- Aprovechamiento económico mediante la generación de energía eléctrica y posterior venta e inyección a la red de suministro eléctrico.

\section{Opciones de aprovechamiento del digestato}

- Aprovechamiento económico mediante su comercialización como fertilizante orgánico de alto valor agregado.

- Aprovechamiento económico mediante su comercialización como mejorador de suelo. 


\section{CONCLUSIONES}

El rezago en el desarrollo tecnológico para dar tratamiento y disposición a los RSU que existe en Nicaragua conlleva grandes consecuencias ambientales y sanitarias. Los proyectos de plantas de DA para dar tratamiento a la FORSU cuentan con gran auge en países europeos debido a un proceso evolutivo en su desarrollo y acumulación de experiencia, en México el proceso de implementación de proyectos de plantas de DA requiere llevar a cabo un aprovechamiento directo de experiencias internacionales en el desarrollo de DA, como la de los países con mayor éxito y presencia de DA para así implementar esta tecnología con el mejor aprovechamiento de tiempo y de recursos.

Los proyectos de plantas de DA presentan una alternativa integral para dar continuidad a la gestión de residuos al dar un aprovechamiento económico y energético a los residuos. Esta tecnología da tratamiento únicamente a la FORSU dejando a un lado la FIRSU; sin embargo genera una situación favorable para dar aprovechamiento a la FIRSU.

Las barreras que enfrentan los proyectos de plantas de DA comprenden diferentes rubros, para poder superar tales barreras es necesario que el modelo de proyectos cambie el enfoque clásico de ser únicamente una alternativa de tratamiento de residuos para darles disposición. El cambio de enfoque que se propone establece que los residuos aún son susceptibles de valoración y genera nuevos modelos de negocio al proponer un aprovechamiento energético y económico de los productos de la DA.

Así también, el desarrollo de estos proyectos requiere cambios en aspectos que potencialmente mantienen en rezago el desarrollo del país, como lo son aspectos sociales de cultura de consumo y generación de residuos, aspectos normativos laxos que mantienen sistemas como los rellenos sanitarios en la principal alternativa de tratamiento y disposición de residuos, políticas débiles en cuanto al apoyo e impulso de transición tecnológica y cuidado al ambiente.

La propuesta de estrategia está dirigida a las municipalidades como Estelí; su implementación se lleva a cabo dentro de las fases de desarrollo de proyectos, Estudio de gran visión y Estudio de pre-factibilidad principalmente, esta estrategia establece un modelo de negocio de proyecto que ofrece entradas económicas para elevar su factibilidad a partir del aprovechamiento de los productos del proceso de DA, establece las alternativas de financiamiento que contribuyen a disminuir el costo de inversión para la entidad federativa y establece acciones para lograr la aceptación social y superación de las barreras técnicas que dificultan el desarrollo de proyectos de plantas de DA. 


\section{REFERENCIAS}

Agencia Andaluza de la Energía. (2011). Estudio Básico del Biogás. Andalucía, España.

Alcaide Tour, A. (2012). Residuos Sólidos Urbanos una consecuencia de la vida. España: Universidad Jaume I.

Arsova, L. (Mayo de 2010). Anaerobic digestion of food waste: Current status, problems and an alternative product. Columbia, New York, United States: Earth Engeeniring Center Columbia University.

Baxter , D., \& Al Seadi, T. (2013). AD of the organic fraction of MSW, System overview for source and central separated waste. Biosantech. BM. (2010). http://datos.bancomundial.org/indicador/AG.CON.FERT.ZS. Banco Mundial.

Borrello, F. (2011). "Tratamiento de residuos y aprovechamiento de biogás para calefacción y generación de energía".

Universidad de Buenos Aires Facultad de Ingeniería. Buenrostro, O. (2006). La producción de Residuos Sólidos Municipales y sus implicaciones ambientales.

California integrated Waste Management Board. (2008). Current Anaerobic Digestion Technologies used for Treatment of Municipal Solid Waste. Sacramento, California, Estados Unidos: CEPA.

Cárdenas Espinosa, R. (17 de 12 de 2010). mailxmail.com. Obtenido de Planificación de proyectos. Enfoque de marco lógico: www.mailxmail.com/curso-planificación-proyectosenfoquemarco-logico/estrategia-proyecto-1-2

Cavinato, C. (2011). Anaerobic Digestion Fundamentals. Venecia.

Centro de investigación y Docencia Económicas, División de Administración Pública. (Enero de

2012). Recuperado el 31 de Octubre de 2016, de http://www.scielo.org. mx/scielo.php?pid=S1405-10792012000100007\&script=sci arttext Chagumaira, I., Fyfield, N., Mangan, A., Roper, Y., Vella, D., \& Wong, J. (2011). Biogas plant proposal. Devikulum, India: Faculty of Engineering and Industrial Sciences. Swinburne University of Technology.

Duran Moreno, A., Garcés Rodríguez, M., Velasco, A., Delgadillo Hernández, N., Moreno Gutiérrez, A., \& Gutiérrez Lara, R. (2013). Mexico city's municipal solid waste characteristics and

composition analysis. México, D.F.

Gonzalez, S. P. (2011). Propuesta tecnológica para la valorización de la fracción orgánica de los

residuos solidos urbanos generados en el Distrito federal. México D.F. H. Hartmann, B. A. (2006). Strategies for the anaerobic digestion of the organic fraction of municipal solid waste: an overview. Water Science \& Technology, Vol 53 (No 8 pp 7-22 Q ). IWA Publishing .

Hagenmeyer, D. o. (2003). Digestión anaerobia seca versus digestión anaerobia húmeda. Pamplona : LINDE-KCA-DRESDEN GMBH.

Hartmann, H., \& Ahring, B. K. (2005). Strategies for the anaerobic digestion of the organic fraction of municipal solid waste. 4th International Symposium on Anaerobic Digestion of Solid Waste, (págs. 34-51). Copenhague, Dinamarca. 
Henry Javier Vílchez Pérez: Es graduado de Ingeniero Civil, con Master en Ingeniería Ambiental del Programa de Investigación Estudios Nacionales y Servicios Ambientales de la Universidad Nacional de Ingeniería. Es profesor de las asignaturas de ingeniería sanitaria y gestión integra de residuos sólidos. Su área de investigación es la disposición final de residuos sólidos y subproductos y sistemas de tratamiento de agua residual. 